\title{
ACIDOSE METABÓLICA POR HIPERGLICEMIA TRANSITÓRIA PÓS ENUCLEAÇÃO DE INSULINOMA PANCREÁTICO
}

\section{Metabolic acidosis secondary to transient hyperglycemia after pancreatic insuli- noma enucleation}

\author{
Franz Robert APODACA-TORREZ, Alberto GOLDENBERG, Sarhan Sydney SAAD, \\ Edson José LOBO, Tarcísio TRIVIÑO
}

ABCDDV/640

\begin{abstract}
Apodaca-Torrez FR, Goldenberg A, Saad SS, Lobo EJ, Triviño T. Acidose metabólica por hiperglicemia transitória pós enucleação de insulinoma pancreático. ABCD Arq Bras Cir Dig 2009;22(1):57-9

RESUMO - Introdução - Insulinoma é a neoplasia endócrina mais frequente dos tumores funcionantes do pâncreas. Origina-se a partir das células beta das ilhotas de Langerhans e caracteriza-se pela produção excessiva de insulina, com consequente hipoglicemia. O tratamento de escolha é a remoção cirúrgica da neoplasia. O presente relato tem como objetivo apresentar uma complicação metabólica pouco observada. Relato do caso - Homem de 41 anos de idade há dois anos com tonturas, visão turva e convulsões. Os sintomas estavam bem relacionados com períodos prolongados de jejum e melhoravam com as refeições, e durante um dos episódios foi constatada a presença de hipoglicemia, melhorando os sintomas imediatamente após administração de glicose endovenosa. A pesquisa glicêmica revelou intensa hipoglicemia. Ultrassonografia, tomografia computadorizada e ressonância magnética de abdome não revelaram nenhum tipo de alterações no pâncreas. Com a hipótese diagnóstica de hiperglicemia orgânica por provável insulinoma, o paciente foi submetido à enucleação da lesão. No $5^{\circ}$ dia do pós-operatório surgiu fístula pancreática e acidose metabólica com resolução satisfatória. O laudo histopatológico mostrou tumor endócrino de pâncreas de $1,5 \mathrm{~cm}$. Conclusão - Todo paciente submetido à ressecção de insulinoma pancreático deve realizar o pós-operatório imediato em unidades de terapia intensiva, monitorando de forma rigorosa os níveis de glicemia como prevenção de acidose metabólica.
\end{abstract}

DESCRITORES - Insulinoma. Neoplasia pancreática. Acidose metabólica

\section{INTRODUÇÃO}

O insulinoma do pâncreas é a neoplasia endócrina funcionante que acomete com maior frequência esta glândula, e origina-se a partir das células beta das ilhotas de Langerhans. O quadro clínico é caracterizado, fundamentalmente, pela presença de sintomas neuroglicopênicos e neurovegetativos ${ }^{8}$. Classicamente, a tríade descrita por Whipple em 1938, se constitui na melhor forma de identificar e diagnosticar esta neoplasia ${ }^{10}$. O tratamento de escolha é o cirúrgico, que consiste na enucleação ou ressecção pancreática, dependendo das características macroscópicas e histológicas do tumor ${ }^{9}$. A fístula pancreática continua sendo a principal complicação deste tipo de tratamento. Outra situação clínica frequentemente observada no pósoperatório imediato destes doentes, é o aparecimento de hiperglicemia transitória em aproximadamente $97 \%$ dos casos $^{11}$, e quadros de diabete melito no pós-operatório tardio em até $30 \%$ das vezes, especialmente em pacientes submetidos à ressecções pancreáticas ${ }^{2}$. São apresentadas

Trabalho realizado na Disciplina de Gastroenterologia Cirúrgica do Departamento de Cirurgia da Universidade Federal de São Paulo - Escola Paulista de Medicina, São Paulo, SP, Brasil.

Endereço de corespondência: Franz R Apodaca-Torreze-mail. apodaca@uol.com.br nesta comunicação, as características clínicas, de imagens e cirúrgicas de uma forma pouco usual de evolução de paciente submetido ao tratamento cirúrgico de insulinoma.

\section{RELATO DE CASO}

Homem de 41 anos de idade com história de dois anos de sintomas caracterizados por tonturas, visão turva e convulsões. Os sintomas estavam bem relacionados com períodos prolongados de jejum e melhoravam com as refeições. Referia ganho de aproximadamente $10 \mathrm{~kg}$ de peso neste período. Estava sendo tratado por neurologista com anticonvulsivantes há um ano. Há seis meses durante um dos episódios, foi constatada a presença de hipoglicemia, melhorando os sintomas imediatamente após administração de glicose endovenosa. A partir deste dado, foi submetido à investigação laboratorial e de imagem. A dosagem de glicemia em jejum foi de $40 \mathrm{mg} / \mathrm{dL}$; da insulina, 36,07 UI (até $25 \mathrm{UI})$; do peptídeo C, $5,94 \mathrm{mg} / \mathrm{mL}(1,0-5,0$ $\mathrm{mg} / \mathrm{mL})$; da pro-insulina, $94,2 \mathrm{pmol} / \mathrm{mL}(7,9-15 \mathrm{pmol} /$ $\mathrm{ml}$ ). A relação insulina/glicemia (índice de Fajans) foi de 0,9 . O teste do jejum prolongado mostrou-se positivo com 180 minutos, sendo interrompido pelo aparecimento de sintomas neuroglicopênicos. Ultra-sonografia, tomografia computadorizada e ressonância magnética de abdome não 
revelaram nenhum tipo de alterações no pâncreas.

Com a hipótese diagnóstica de hiperglicemia orgânica por provável insulinoma do pâncreas, o paciente foi submetido a procedimento cirúrgico. A abordagem foi feita através de incisão subcostal bilateral. Após a abertura da retrocavidade e exposição da glândula pancreática em toda sua extensão, foi localizado na face anterior da transição corpo-cauda, nódulo de cor vinhosa, consistência fibroelástica de aproximadamente $1,5 \mathrm{~cm}$ de diâmetro. Optou-se pela enucleação da lesão (Figura 1) e drenagem da cavidade com dreno túbulo laminar.

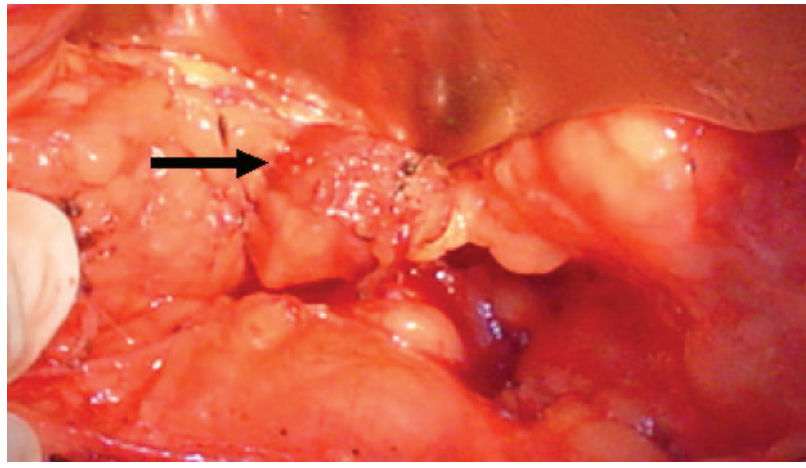

FIGURA 1 - Insulinoma localizado na borda inferior da transição corpo-caudal do pâncreas (seta)

Foi monitorada a glicemia durante o ato operatório. No final da operação o valor da glicemia foi de $220 \mathrm{mg} /$ dL. Após duas horas em sala de recuperação anestésica, o paciente foi transferido para a enfermaria. Seis horas após, apresentou quadro clínico caracterizado por taquipnéia, sudorese e confusão mental, dosagem de glicemia capilar evidenciou HI (valores acima de $400 \mathrm{mg} / \mathrm{dL}$ ). Gasometria arterial com $\mathrm{pH}$ de 7,32 e BE -5. Imediatamente foram administradas 10 UI de insulina regular por via subcutânea e hidratação rápida com $1000 \mathrm{~mL}$ de ringer-lactato. Ele foi transferido para unidade de cuidados intensivos, monitorização da curva glicêmica e execução de exames laboratoriais com os seguintes resultados: hemoglobina $18,1 \mathrm{~g} / \mathrm{dL}$; hematócrito $51 \%$; leucócitos $20.900 \mathrm{~mm}^{3}$ (0/96); plaquetas $282.000 \mathrm{~mm}^{3}$; creatinina $1,0 \mathrm{mg} / \mathrm{dL}$; uréia $35 \mathrm{mg} / \mathrm{dL}$; Na134,4 mEq/L; potásio $3,4 \mathrm{mEq} / \mathrm{L} ; \mathrm{Mg}$ $1,6 \mathrm{mEq} / \mathrm{L}$; amilase $214 \mathrm{UI}$; TGO $76 \mathrm{U}$; TGP 41 U. Oito horas após, o doente apresentava-se com glicemia de 198 $\mathrm{mg} / \mathrm{dL}$ e reversão dos sintomas anteriormente referidos. No segundo dia de pós-operatório apresentou glicemia de $195 \mathrm{mg} / \mathrm{dL}$, e transferido para enfermaria. No $5^{\circ}$ foi feito diagnóstico fístula pancreática, dosagem de amilase do dreno acima de 1000 UI. Iniciada antibioticoterapia com ceftriaxona dissódica e metronidazol.

Os níveis glicêmicos se mantiveram dentro dos parâmetros da normalidade a partir do $7^{\circ}$ dia de pós-operatório. Recebeu alta hospitalar no $35^{\circ}$ dia. O laudo histopatológico mostrou tumor endócrino de pâncreas de $1,5 \mathrm{~cm}$ de diâmetro (Figura 2), índice de mitose de 3 por 10 campos. A imunohistoquímica foi positiva para sinaptofisina, cromogranina e enolase, sendo negativa para o Ps. O Ki-67 mostrou-se inconclusivo. Atualmente paciente encontra-se em pós-operatório de médio prazo, evoluindo com níveis de glicemia dentro da normalidade $(98 \mathrm{mg} / \mathrm{dL})$ e sem nenhum tipo de medicação.

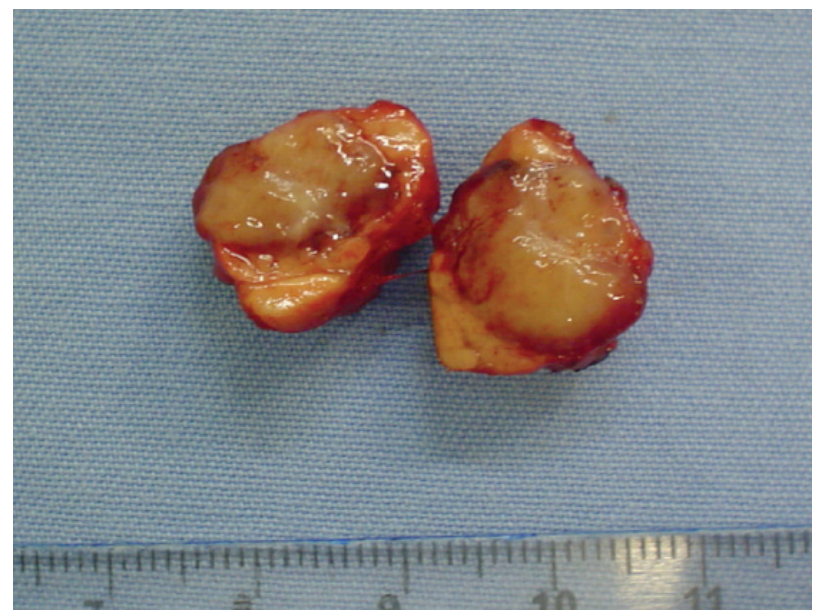

FIGURA 2 - Aspecto macroscópico do insulinoma (seccionado)

\section{DISCUSSÃO}

Durante muitos anos somente o quadro clínico associado à constatação laboratorial da hipoglicemia e hiperinsulinemia, foram os métodos utilizados para a indicação cirúrgica dos insulinomas pancreáticos ${ }^{2}$. Os índices de diagnóstico falso-positivos não eram desprezíveis, aumentando assim as pancreatectomias às cegas hoje em dia abandonadas 9 . Os métodos de radioimunoensaio para dosagem de insulina, do peptídeo $\mathrm{C}$ e da pro-insulina, melhoraram consideravelmente o diagnóstico. Entretanto, a localização topográfica da lesão continuava a ser um problema a ser resolvido6. Com o progresso tecnológico, os métodos de imagem elevaram a acurácia diagnóstica da localização dos insulinomas. Foi a utilização da ultrassonografia intra-operatória associada à palpação pancreática que conseguiram índices de até $100 \%$ na localização destas neoplasias durante a operação ${ }^{7}$. No caso em discussão, a neoplasia foi localizada facilmente após a ampla exposição e palpação intra-operatória do pâncreas, embora não foram utilizados métodos de imagem pré-operatórios mais acurados como a tomografia multi-slice e a ecoendoscopia.

A ressecção cirúrgica destas neoplasias - por enucleação ou por pancreatectomia -, constitui- se na melhor alternativa de cura definitiva. Ela dependerá da localização, do número, do tamanho, da relação com o ducto pancreático principal e da natureza histopatológica do tumor ${ }^{8}$. Este tipo de abordagem pode gerar uma série de complicações, das quais a fístula pancreática é a mais observada e de evolução favorável na maioria dos casos ${ }^{2}$. Outras complicações são os abscessos peripancreáticos, as hemorragias e a pancreatite aguda ${ }^{7,8,9}$. Neste paciente teve-se a oportunidade de observar complicações decorrentes da fístula pancreática, evoluindo posteriormente com abscesso peripancreático, situação que motivou dois novos procedimentos cirúrgicos para drenagem, prolongando assim o período de internação.

Dentre as complicações metabólicas, a hiperglice- 
mia transitória é a ocorrência clínica mais comumente observada $^{11}$. No entanto, sua regularização acontece nas primeiras semanas e geralmente é de fácil controle com a utilização esporádica de insulina regular, não produzindo grandes repercussões clínicas na evolução destes doentes $^{2,11}$. Uma situação de aparecimento mais tardio é o diabete melito, o qual pode incidir entre 4 a $33 \%$ dos pacientes e está relacionado aqueles que foram submetidos a pancreatectomia ${ }^{3}$. Seu controle clínico não costuma oferecer grandes dificuldades.

O estado hiperosmolar secundário à hiperglicemia ou cetoacidose diabética, é complicação aguda grave, com mortalidade de aproximadamente $5 \%$ em centros especializados. É geralmente observado em pacientes com diagnóstico de diabete melito tipo I descompensado, resultado do déficit absoluto ou parcial de insulina e desencadeado por uso de medicamentos ou desordens dietéticas ${ }^{4}$. Esta emergência clínica está descrita já com níveis de glicemia acima de $250 \mathrm{mg} / \mathrm{dL}$ e seu tratamento resume-se na hidratação, oxigenação e controle dos níveis glicêmicos com insulina ${ }^{6}$. No paciente com insulinoma pancreático onde os níveis de insulina estão aumentados, existe diminuição da produção dos hormônios hiperglicemiantes $^{5}$. A hiperglicemia transitória observada logo após a ressecção do insulinoma, estaria relacionada com a interrupção abrupta da hipersecreção de insulina produzida pelo tumor, ocasionando assim aumento de hormônios (glucagon, cortisol e hormônio do crescimento) e substâncias hiperglicemiantes (catecolaminas). Produzem-se, assim, distúrbios no metabolismo dos carbohidratos, proteínas e lipídeos, levando a quadros de hiperglicemia passageira semelhantes aos observados na cetoacidose diabética9 e raramente com o quadro intenso de acidose aqui relatado.

\section{CONCLUSÃO}

Recomenda-se que todo paciente submetido à ressecção de insulinoma pancreático, deva realizar o pós-operatório imediato em unidades de terapia intensiva, monitorandose de forma rigorosa os níveis de glicemia, especialmente nas primeiras horas.

Apodaca-Torrez FR, Goldenberg A, Saad SS, Lobo EJ, Triviño T. Metabolic acidosis secondary to transient hyperglycemia after pancreatic insulinoma enucleation. ABCD Arq Bras Cir Dig 2009;22(1):57-9

ABSTRACT - Introduction - The insulinoma is the most frequent endocrine neoplasm among the functional pancreatic tumors. It originates in the beta cells of the islets of Langerhans and is characterized by the oversecretion of insulin, leading to hypoglycemia. The treatment of choice is the surgical excision of the tumor. The aim of the present report is to describe a rarely observed metabolic complication. Case report - The case is presented of a 41-year-old man with a 2-year history of dizziness, blurred vision and seizures. The symptoms were closely related to prolonged fasting and improved with eating; hypoglycemia was found during one of the episodes. Symptoms were relieved immediately after intravenous administration of glucose. Blood glucose workup showed severe hypoglycemia. Abdominal ultrasonography, computed tomography and magnetic resonance imaging did not show any alteration in the pancreas. With the diagnostic hypothesis of organic hypoglycemia from a likely insulinoma, the patient underwent the enucleation of the lesion. On the 5th postoperative day, a pancreatic fistula appeared, as well as metabolic acidosis which resolved satisfactorily. The histopathological report showed a $1.5-\mathrm{cm}$ endocrine pancreatic tumor. Conclusion-Every patient submitted to pancreatic insulinoma resection should stay in an intensive care unit during the immediate postoperative period and their glycemic levels must be monitored closely to prevent metabolic acidosis.

HEADING - Insulinoma. Pancreatic neoplasms. Metabolic acidosis.

\section{REFERÊNCIAS}

1. Abboud B, Boujaoude J. Occult sporadic insulinoma: Localization and surgical strategy. World J Gastroenterol 2008; 14(5): 657-665.

2. Apodaca-Torrez FR, Triviño T, Lobo EJ, Goldenberg A, Benvenuto MR, Ardengh JC. Insulinoma de pancreas. Cir Esp 2006;80(1):3-8.

3. Boukhman MP, Karam JH, Shaver J, Siperstein AE, Quan-Yang Duh, Clark OH. Insulinoma- Experience from 1950 to 1995. West J Med 1998; 169:98-104.

4. Foss-Freitas MC, Foss MC. Cetoacidose Diabética e Estado Hiperglicêmico Hiperosmolar. Medicina, Ribeirão Preto 2003; 36: 389-393.

5. Glaser B, Valtysson G, Fajans SS, Vinik AI, Cho K, Thompson N. Gastrointestinal / Pancreatic Hormone Concentrations in the Portal Venous System of Nine Patients With Organic Hyperinsulinism. Metabolism 1981; 30(10): 1001-1010.

6. Israel RS. Diabetic ketoacidosis. Emerg Med Clin North Am 1989; 7: 859-871.
7. Ravi K, Britton B. Surgical approach to insulinomas: are pre-operative localisation tests necessary?. Ann R Coll Surg Engl 2007; 89: 212-217.

8. Stefanini P, Carboni M, Patrassi N, Basoli A. Beta islet cell tumours of the pancreas: results of a study of 1067 cases. Surgery 1974; 75: 597-609.

9. Tucker ON, Crotty PL, Conlon KC. The management of insulinoma. Br J Surg 2006; 93: 264-275.

10. Whipple AO. The surgical therapy of hyperinsulinism. J Int Chir 1938; 3:237276.

11. Xi Chen, Wei-Yao Cai, Wei-Ping Yang, Hong-Wei Li. Pancreatic Insulinomas: diagnosis and surgical treatment of 74 patients. HBPD Int 2002; 1:458-461.

Fonte de financiamento: não há Conflito de interesse: não há Recebido para publicação: 02/08/2008 Aceito para publicação: 14/12/2008 